\title{
Letter \\ Visualizing multiple organ failure: a method for analyzing temporal and dynamic relations between failing systems and interventions
}

\author{
Yusuf Alper Kılıç, Kaya Yorgancı and İskender Sayek \\ Hacettepe University School of Medicine, Department of General Surgery, Ankara, Turkey
}

Corresponding author: Yusuf Alper Kılıç, yusufa@hacettepe.edu.tr

Published: 6 July 2007

This article is online at http://ccforum.com/content/11/4/417

(c) 2007 BioMed Central Ltd

Multiple organ failure is common among critically ill patients who have a prolonged stay in the intensive care unit or die. Apart from causing significant morbidity and mortality, the treatment for multiple organ failure requires use of limited and costly resources. Although the epidemiology and progression of multiple organ failure are well described, it is not possible to conduct detailed and reliable analyses of the temporal relations between failing organs and the effects of interventions (for instance, institution of antibiotic therapy or surgery). In addition, progression of multiple organ failure may differ between patient populations [1]. We describe a method that allows evaluation of the temporal and dynamic nature of multiple organ failure.

We developed this approach by using multiple organ failure scores (Multiple Organ Dysfunction Score [MODS], Logistic Organ Dysfunction Score [LODS] and Sequential Organ Failure Assessment [SOFA]) stored in our critical care database [2-5]. Using these scores, we have constructed a surface topogram of organ failure for the entire stay in the intensive care unit, using the open source data visualization software OpenDX Visualization Data Explorer (version 4.4.4) [6].

The software outputs a graphic in which each organ system is represented by a row, and the various degrees of organ failure are represented by different colours ranging (in order of severity) from blue, green and yellow to red (Figure 1). This allows clear and rapid visual identification of changes in organ systems, temporal relations within and between failing organs, and effects of interventions. In most patients in whom disease severity is low, the software outputs a graphic that resembles a smooth sea surface (all blue). In patients with severe disease the graphic resembles a 'stormy sea', which ends with a 'tsunami'. In the given example, exacerbation of organ failure by surgical intervention and relief with abdominal decompression can clearly be identified. Also, worsening and correction of renal function follows parallel changes in cardiac function.
Critical Care 2007, 11:417 (doi:10.1186/cc5941)

Figure 1

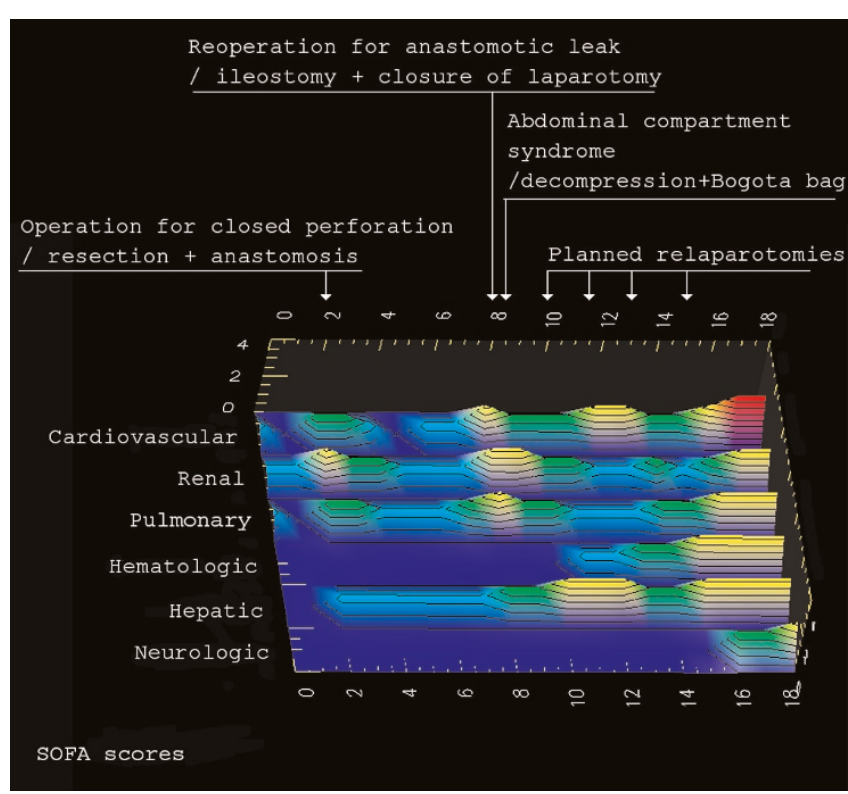

Graphical representation of SOFA scores. Shown is a graphical representation of the evolution over time in Sequential Organ Failure Assessment (SOFA) scores: a 'stormy' intensive care unit course ending with a 'tsunami'.

This approach can also be used to conduct quality assessment studies, to evaluate intensive care unit performance and patient safety, and to communicate complicated relations between data arrays. The method is also useful for visualizing concordance and calibration between different organ failure systems (Figure 2). In the second example, compared with MODS and SOFA systems, the LODS system appears to underestimate cardiovascular failure.

In conclusion, we believe that this method will be useful in analyzing temporal and dynamic relations within and between 


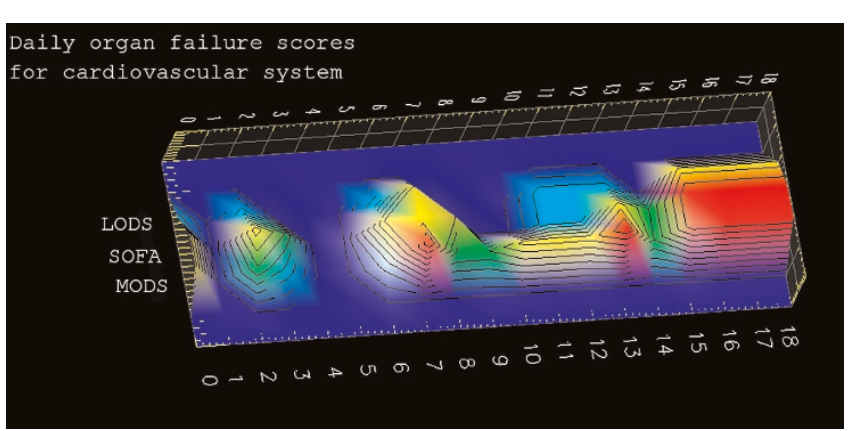

Graphical representation of the performance of LOD, SOFA and MODS: cardiovascular system. Shown are data of another patient, illustrating differences between organ failure scores in assessing the cardiovascular system. LODS, Logistic Organ Dysfunction Score; MODS, Multiple Organ Dysfunction Score; SOFA, Sequential Organ Failure Assessment.

failing organ systems and related interventions. We also suggest that this approach could be used as a basis for constructing statistical methods to analyze these relations quantitatively.

\section{Competing interests}

YAK is the author and director of the Muavenet Intensive Care Information System, which is an open access, online academic information system. The other authors declare that they have no competing interests.

\section{References}

1. Kilic YA, Dogrul AB, Karakoc D, Yildiz B, Yorganci K, Sayek I: Impact of organ failure on mortality prediction in a Turkish surgical intensive care unit. Intensive Care Med 2005, Suppl 1: S47

2. Marshall JC, Cook DJ, Christou NV, Bernard GR, Sprung CL, Sibbald WJ: Multiple organ dysfunction score: a reliable descriptor of a complex clinical outcome. Crit Care Med 1995, 23:1638-1652.

3. Vincent JL, Moreno R, Takala J, Willatts S, De Mendonca A, Bruining H, Reinhart CK, Suter PM, Thijs LG: The SOFA (Sepsisrelated Organ Failure Assessment) score to describe organ dysfunction/failure. On behalf of the Working Group on Sepsis-Related Problems of the European Society of Intensive Care Medicine. Intensive Care Med 1996, 22:707-710.

4. Le Gall JR, Klar J, Lemeshow S, Saulnier F, Alberti C, Artigas A, Teres D: The Logistic Organ Dysfunction System. A new way to assess organ dysfunction in the intensive care unit. JAMA 1996, 276:802-810

5. Muavenet Intensive Care Information System [http://www.icu. hacettepe.edu.tr/micis.html]

6. OpenDX [http://www.opendx.org] 